January 2011

\title{
Comparison of predictive protocols in chest pain patients in a tertiary care hospital in Karachi, Pakistan
}

\author{
Omer Moin \\ Aga Khan University \\ Uzma Khan \\ Aga Khan University \\ Nadeem Ullah Khan \\ Aga khan University, nadeemullah.khan@aku.edu \\ Munawar Khursheed \\ Aga Khan University \\ Junaid Razzak \\ Aga Khan University, junaid.razzak@aku.edu
}

Follow this and additional works at: http://ecommons.aku.edu/pakistan_fhs_mc_emerg_med Part of the Cardiology Commons, and the Cardiovascular Diseases Commons

\section{Recommended Citation}

Moin, O., Khan, U., Khan, N., Khursheed, M., Razzak, J. (2011). Comparison of predictive protocols in chest pain patients in a tertiary care hospital in Karachi, Pakistan. The Internet Journal of Emergency Medicine, 6(2), 1-6.

Available at: http://ecommons.aku.edu/pakistan_fhs_mc_emerg_med/93 


\title{
Comparison of Predictive Protocols in Chest Pain Patients in a Tertiary Care Hospital in Karachi, Pakistan
}

\author{
O Moin, U Khan, N Khan, M Khursheed, J Razzak
}

\begin{abstract}
Citation
O Moin, U Khan, N Khan, M Khursheed, J Razzak. Comparison of Predictive Protocols in Chest Pain Patients in a Tertiary Care Hospital in Karachi, Pakistan. The Internet Journal of Emergency Medicine. 2009 Volume 6 Number 2.
\end{abstract}

\begin{abstract}
Background: The most challenging aspect in evaluation of a patient presenting with chest pain in the Emergency Department (ED) is diagnosis of acute cardiac ischemia (ACI). Various decisions aids have been developed to aid the ED physician in the diagnosis of chest pain. Three different predictive protocols - the Goldman protocol, ACI-TIPI, and the Troponin protocol are used in predicting the occurrence of $\mathrm{ACl}$. None of these decision aids have been tested in the Pakistani population, where risk factors and prevalence of CAD are much different than what is seen in other regions of the world.Aims: The aim of this study was to compare how three different predictive protocols for ACl fared in the Pakistani population.Methods:This retrospective case series included a consecutive sample of 212 patients who presented to the ED with symptoms of chest pain or its equivalent. Patient data was collected and retrospectively analyzed with each predictive protocol to analyze sensitivity, specificity, PPV, and NPV of each protocol.Results: $63 \%$ of patients were diagnosed with ACl. The sensitivities of the Goldman, ACI-TIPI, and Troponin protocols were 94\%, 98\%, and 38\% respectively. The specificities of the Goldman, ACI-TIPI, and Troponin protocols were $14 \%, 29 \%$, and $95 \%$ respectively. Conclusion: Sensitivities from a small sample size show promise in the use of predictive protocols for chest pain in the Pakistani population. However, prospective studies on a larger level need to be conducted to validate these findings.
\end{abstract}

\section{INTRODUCTION}

Chest pain is a common presentation to the emergency department. The evaluation of patients presenting with chest pain has proven to be a difficult task for emergency physicians. The most challenging aspect in evaluation of chest pain is diagnosis of acute cardiac ischemia (ACI). Failure to identify patients with ACI results in serious consequences. 2 to $5 \%$ of patients with ACI are discharged from the emergency department and are at subsequent risk of having a mortality and morbidity that is twice those who are correctly diagnosed and admitted [1]. On the other hand admission of patients who are at low risk of ACI leads to unnecessary admissions and hospital expenditures. In the West, of the $50-70 \%$ of patients who are admitted in hospitals for complaints of chest pain, only $25-30 \%$ actually have a diagnosis of ACI [2].

Various decisions aids have been developed to aid the ED physician in the diagnosis of chest pain. These protocols have widely been tested and tried in developed countries and have shown to be useful in the triage of patients presenting with chest pain. However no single clinical pathway or predictive instrument has triumphed above others in proving to be both easy to use and provide a means by which the diagnosis made consistently outperforms and improves the physician's decision [3]. The clinical impact of these decision aids has remained low, and despite large multicenter studies demonstrating their potential, these decision aids have still yet to be put into widespread clinical use [4].

Of the available decision aids for evaluation of chest pain this study focuses on three protocols that have been developed to help in the diagnosis of ACI. The Goldman Chest Pain protocol [5] helps identify patients with chest pain of having specifically an acute myocardial infarction. The Acute Cardiac Ischemia Time-Insensitive Predictive Instrument (ACI-TIPI) [6] uses a computerized prediction to give the probability of a patient having acute ischemia. Troponins (cTnT and cTnI) are myocardial regulatory proteins that help accelerate the diagnosis of ACI because of their cardiac specificity and elevated levels being an independent risk factor for ACI [2].

None of these decision aids have been tested in Pakistan - 
where the prevalence of coronary artery disease is unknown and where risk factors of ACI differ from other regions of the world. It is estimated that one in four middle aged adults have prevalent coronary artery disease in Pakistan with women being at greater risk [7]. This affected population is also found to have established risk factors such as hypertension, dyslipidemia, and diabetes and also have a positive family history of coronary artery disease. Various studies of Pakistani's living outside of Pakistan have substantiated these claims. South Asians in North America have shown to have the highest mortality rates of any other ethnic group and are also at an increased risk of atherosclerosis not attributed to conventional risk factors [8]. With these facts and figures, it is important to assess whether protocols used to diagnose ACI in patients with chest pain in developed countries, where the populations have different characteristics and risk factors, have any yield on the Pakistani population.

In this study we aimed to use three different protocols to evaluate patients who presented with chest pain to the emergency department. We undertook the study to better assess which of these decision aids would be best suited for triage of patients presenting with chest pain in the Pakistani population.

\section{METHODOLOGY}

Study Design: Retrospective case series of 64 adult patients.

Study Setting: The Aga Khan University Hospital (AKUH) is a 675 bedded tertiary care teaching hospital in Karachi. The ED at AKUH sees approximately 45000 patients per year. Patients were enrolled during a study period from September 2007 to February 2008.

Study Approval: Study approval was secured from the Ethics Review Committee at the Aga Khan University in

Karachi, Pakistan.

Study Population: Patients that presented to the ED of AKUH with a primary complaint of chest pain or its equivalent (i.e. left arm pain or shortness of breath).

Inclusion Criteria: All adult patients more than 18 years of age presenting to emergency department with non-traumatic chest pain/discomfort, or chest pain equivalent (i.e. left arm pain and shortness of breath) were included. Patients were required to give their consent to take part in the study and consent was taken during the patient presentation to the ED.
Exclusion Criteria: All patients not giving consent were excluded from the study as well as patients with traumatic injury or those too critically ill to be able to answer study questions were also excluded from the study.

Data Analysis: Data from the patient's file including demographic information, clinical characteristics, risk factors, ECG findings, troponin results and other variables used in included protocols was recorded by a research volunteer. The outcome measure was to see if a patient had developed ACI. To standardize the results we used a final diagnosis of ACI by the cardiology service as being evidence of the patient having ACI. Data was analyzed on SPSS for frequencies of demographic and clinical variables. Sensitivity, specificity, positive predictive value (PPV), and negative predictive value (NPV) of chest pain protocols were calculated using standard computer calculators.

\section{RESULTS}

During the study period a selection of 212 consecutive patients that presented to the ED, fulfilled the inclusion criteria, and gave consent were selected. 77 patients were excluded from the study because their files had insufficient data or no final diagnosis. Of the 135 remaining patients, 64 patients had an ED diagnosis of ACI whereas 71 had an ED diagnosis of not having ACI. Both of these groups were allowed further testing for confirmation of ACI by the cardiology service. The cardiology service carried out further testing for confirmation of ACI on 64 patients from both the ED diagnosis of ACI group and ED Diagnosis of not having ACI group. These 64 patients on whom further testing was done were used in our study. (see Figure 1)

We collected and compared data on the 64 patients that were used in our study. Demographic data is summarized in Table 1. Of the total study population 40 patients $(63 \%)$ had a final diagnosis of ACI versus 20 patients (37\%) that did not. There were 3 reported deaths in the ACI diagnosed group and 1 reported death in the non ACI group.

The mean age for patients diagnosed with ACI was 60 compared to a mean age of 56 for patients not diagnosed with ACI. There was also a difference seen in the gender break up in the two groups - the ACI group having 70\% males and $30 \%$ females whereas the non-ACI group saw an even break up (50\% each).

The chief complaints of patients eventually diagnosed with $\mathrm{ACI}$ are chest pain (95\%), a pain pressing in nature (75\%), shortness of breath (58\%), and diaphoresis (38\%). Other 
chief complaints were pain radiating to the left arm or left shoulder. Surprisingly the presenting complaints were found to be similar in frequency of both patients diagnosed with ACI and not having ACI, further delineating the difficulty in diagnosis.

Predictive protocols were used retrospectively to evaluate their predictive powers in the diagnosis and exclusion of ACI. The Goldman protocol fared well in terms of sensitivity (94\%) but this came at the expense of increasing the number of false positives which resulted in it having a specificity of $14 \%$. In contrast the ACI-TIPI protocol had a higher sensitivity and specificity, $98 \%$ and $29 \%$ respectively. Both the Goldman and ACI-TIPI protocols had very similar positive predictive values ( $64 \%$ and $71 \%$ respectively) but the Goldman protocol had a much higher negative predictive value ( $40 \%$ and $14 \%$ respectively). (see Table 2 )

When the Troponin protocol was used it was found that it was of little diagnostic value in terms of sensitivity. Initial Troponin I done on arrival to the ED was only found to be elevated in $38 \%$ of the patients diagnosed with ACI.

However, troponins as expected had a high specificity $-95 \%$ in our study.

\section{Figure 1}

Figure 1 - Patient Selection: Figure details patient selection for study

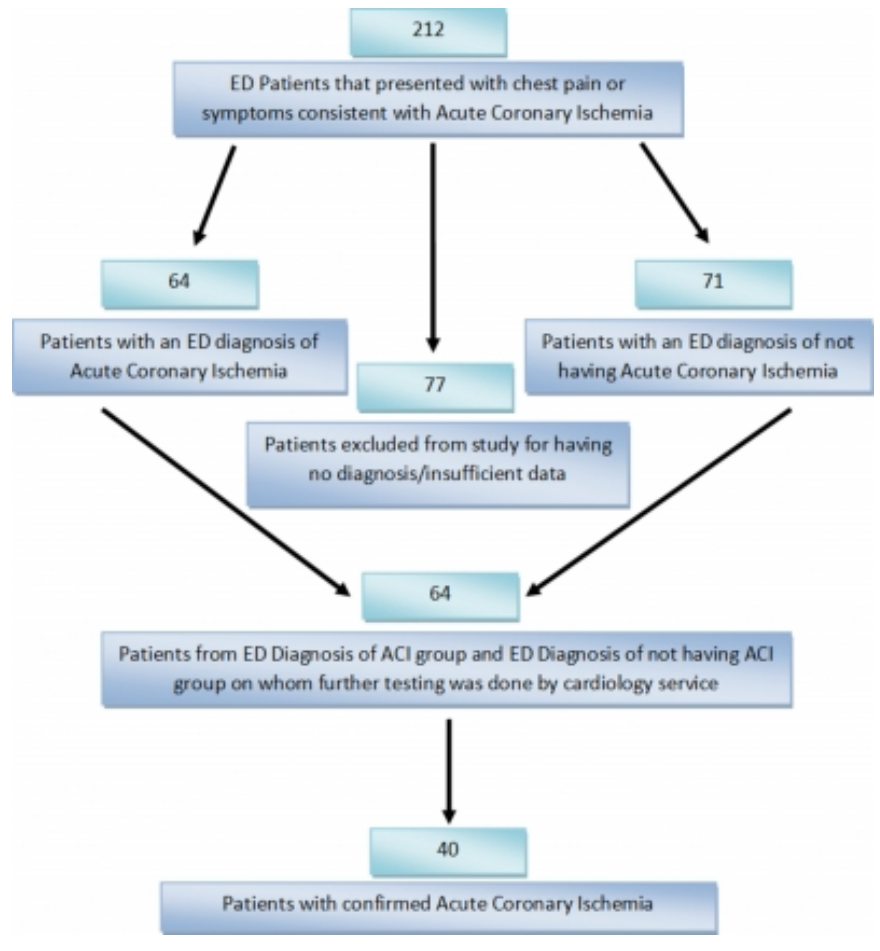

Figure 2

Table 1 - Demographics of Patients

\begin{tabular}{|c|c|c|}
\hline Variable & $\operatorname{ACI}(n=40)$ & No $A C I(n=24)$ \\
\hline \multicolumn{3}{|l|}{ Present History } \\
\hline Age (y) & 60 (SD 12) & $56(\mathrm{SD} 13)$ \\
\hline \multicolumn{3}{|l|}{ Sex } \\
\hline Men & $28(70 \%)$ & $12(50 \%)$ \\
\hline Women & $12(30 \%)$ & $16(40 \%)$ \\
\hline Chest Pain & $38(95 \%)$ & $24(100 \%)$ \\
\hline Left Am Pain & $8(20 \%)$ & $4 \quad(17 \%)$ \\
\hline Pain Pressing in Nature & $30(75 \%)$ & $18(75 \%)$ \\
\hline Shortness of Breath & $23(58 \%)$ & $13(54 \%)$ \\
\hline Diaphoresis & $15(38 \%)$ & $8 \quad(33 \%)$ \\
\hline Nausea/Vomiting & $11(28 \%)$ & $7 \quad(29 \%)$ \\
\hline Dizziness & $4(10 \%)$ & $3 \quad(13 \%)$ \\
\hline \multicolumn{3}{|l|}{ Past History } \\
\hline Diabetes Mellitus & $16(40 \%)$ & $12(50 \%)$ \\
\hline Hypertension & $26(65 \%)$ & $14(58 \%)$ \\
\hline Dyslipidemia & $10(25 \%)$ & $7 \quad(29 \%)$ \\
\hline Congestive Heart Failure & $5(13 \%)$ & $5 \quad(21 \%)$ \\
\hline Myocardial Infarction & $16(40 \%)$ & $7 \quad(29 \%)$ \\
\hline Family history of Coronary Artery Disease & $7(18 \%)$ & $11(46 \%)$ \\
\hline Smoking & $9 \quad(23 \%)$ & $3(13 \%)$ \\
\hline \multicolumn{3}{|l|}{ ECG } \\
\hline Q-wave & $25(63 \%)$ & $14(58 \%)$ \\
\hline ST-segment elevation & $11(48 \%)$ & $2(8 \%)$ \\
\hline ST-segment depression & $15(38 \%)$ & $5 \quad(21 \%)$ \\
\hline T-wave inversion & $19(48 \%)$ & $17(71 \%)$ \\
\hline Hyper acute $T$ waves & $17(43 \%)$ & $3 \quad(13 \%)$ \\
\hline Left bundle branch block & $2(5 \%)$ & $0 \quad(0 \%)$ \\
\hline \multicolumn{3}{|l|}{ Chemical Markers } \\
\hline Troponin - I & $9 \quad(23 \%)$ & $1 \quad(4 \%)$ \\
\hline CK-MB & $5 \quad(13 \%)$ & $3(13 \%)$ \\
\hline \multicolumn{3}{|l|}{ Outcome } \\
\hline Cardiac Arrest/Heart Failure & $1 \quad(3 \%)$ & $0 \quad(0 \%)$ \\
\hline Death & $3(8 \%)$ & $1 \quad(4 \%)$ \\
\hline
\end{tabular}

\section{Figure 3}

Table 2 - Analysis of Protocols

\begin{tabular}{|c|c|c|c|c|}
\hline Method & Sensitivity & Specificity & $\underline{\text { PPV }}$ & NPV \\
\hline Goldman & $94 \%$ & $14 \%$ & $64 \%$ & $40 \%$ \\
\hline ACI - TIPI & $98 \%$ & $29 \%$ & $71 \%$ & $14 \%$ \\
\hline Tropenins & $35 \%$ & $99 \%$ & $90 \%$ & $54 \%$ \\
\hline
\end{tabular}

\section{DISCUSSION}

The Goldman, ACI TIPI, and Troponin protocols were established to help predict and assist in the diagnosis of ACI. Although not perfect, these protocols have been shown to aid physicians in their clinical evaluation of ACI [9].

Sensitivities of the protocols used in this study are comparable to those reported in other studies. However, our calculated specificities for protocols are lower than those seen in other studies.

The ACI-TIPI protocol is reported by studies to have a sensitivity of $86-95 \%$ and specificity between $78-92 \%$ [10]. This study yielded a sensitivity of $98 \%$ in diagnosing ACI when using the ACI-TIPI protocol, which is comparable to the data present in literature. However the specificity of $29 \%$ while using the ACI-TIPI protocol in this study found to be 
quite low.

The Goldman protocol is used to diagnose only acute myocardial infarction and literature reports it having sensitivity between $88-91 \%$ and a specificity between 70-74\% [10]. Our study reported a higher sensitivity (94\%) when using the Goldman protocol but a much lower specificity of $14 \%$.

The literature reports that when Troponin-I is used in a single presentation to diagnose ACI it is found to have a sensitivity of $39 \%$ with a $95 \%$ Confidence Interval (CI) between $10-78 \%$ and a specificity of $93 \%$ with a $95 \%$ CI between $88-97 \%$. [10] When used in this study troponin I was found to have a comparable sensitivity $(38 \%)$ and specificity $(95 \%)$.

Even with the limited data available for this study it is clear that the protocols used are useful in diagnosing an acute coronary event in patients presenting to the ED and along with the physicians clinical diagnosis can be used to help predict the likelihood of ACI in patients presenting with chest pain.

Another key point to consider in this study is the use of these protocols in the Pakistani population, where studies like this one have not been conducted. It is important that these protocols be prospectively validated in separate populations to ensure their validity and stability [9]. Research is beginning to elucidate that the Pakistani and South Asian population are at considerably greater risk than other races for coronary artery disease. Risk factors for coronary artery disease are also more evident in South Asians, including both conventional and other factors such as genetic predisposition and metabolic syndrome. This study validates the position that many patients who present with ACI have established risk factors before presentation. It also shows that presentation and risk factors in the Pakistani population are not always helpful clues in diagnosing ACI as many patients who didn't have ACI are seen to have very similar presentations and risk factors to those patients that eventually were diagnosed as having an ACI.

\section{LIMITATIONS}

Due to the circumstances involving data collection, our study has several limitations. The data was collected in a retrospective manner (on review of patient files) and thorough data of each patient was not always available. Since we only studied how the predictive protocols would perform retrospectively we did not take into effect the influence that the physician would have on the diagnosis and the obvious outcome change that influence might bring. None of the protocols were tested prospectively during patient presentation to the ED and thus the true sensitivity and specificity of each of the protocols could not be calculated.

Our sample size was also small and may not be representative of the general population. Both the group diagnosed with ACI and the group diagnosed with not having ACI were seen to have very similar characteristics in terms of symptoms and past history and this could be representative of a small sample size.

\section{CONCLUSION}

Predictive protocols for ACI are of useful clinical aid to physicians. In our study on a small sample size we have

found to have calculated sensitivities that are comparable to those reported in literature While this shows promise in the use of predictable protocols for chest pain, we believe that more prospective studies be done on this matter in a larger Pakistani population size, so as to better judge which clinical aids will prove better in this population.

\section{LIST OF ABBREVIATIONS}

ED - Emergency Department

ACI - Acute Coronary Ischemia

ACI-TIPI - Acute Cardiac Ischemia Time-Insensitive Predictive Instrument

AKUH - Aga Khan University Hospital

PPV - Positive Predictive Value

NPV - Negative Predictive Value

\section{References}

1. McCarthy BD, Beshansky JR, D'Agostino RB et al. Missed diagnoses of acute myocardial infarction in the emergency department: results from a multicenter study. Ann Emerg Med 1993; 22:579-582.

2. DeFilippi CR, Runge MS. Evaluating the Chest Pain Patient. Scope of the Problem. Cardiol Clin 1993;17(2):307-26.

3. Kennedy RL, Harrison RF, Marshall SJ. Do we need computer-based decision support for the diagnosis of acute chest pain: discussion paper. J R Soc Med 1993;86:31-34.

4. Lai F, Macmillan J, Daudelin DH et al. The Potential of Training to Increase Acceptance and Use of Computerized Decision Support Systems for Medical Diagnosis. Hum Factors 2006; 48(1):95-108.

5. Goldman L, Cook EF, Brand DA et al. A Computer Protocol to Predict Myocardial Infarction in Emergency Department Patients with Chest Pain. N Engl J Med 1988;318(13):797-803.

6. Selker HP, Beshansky JR, Griffith JL. Use of the Acute Cardiac Ischemia Time-insensitive Predictive Instrument (ACI-TIPI) to Assist with Triage of Patients with Chest Pain 
or Other Symptoms Suggestive of Acute Cardiac Ischemia. A Multicenter, Controlled Clinical Trial. Ann Intern Med 1998;129(11):845-55.

7. Jafar TH, Qadri Z, Chaturvedi N. Coronary Artery

Disease Epidemic in Pakistan: More Electrocardiographic Evidence of Ischemia in Women than in Men. Heart 2008;94(4):408-13.

8. Bainey KR, Jugdutt BI. Increased Burden of Coronary Artery Disease in South-Asians Living in North America. Need for an Aggressive Management Algorithm.
Atherosclerosis 2009;204(1):1-10.

9. Miller CD, Lindsell CJ, Anantharaman V et al. Performance of a Population-based Cardiac Risk

Stratification Tool in Asian Patients with Chest Pain. Acad Emerg Med 2005;12(5):423-30.

10. Lau J, Ioannidis JP, Balk EM et al. Diagnosing Acute Cardiac Ischemia in the Emergency Department: a Systematic Review of the Accuracy and Clinical Effect of Current Technologies. Ann Emerg Med 2001, 37(5):453-60. 


\section{Author Information}

\section{Omer Moin}

Medical College, Aga Khan University

\section{Uzma Khan}

Department of Emergency Medicine, Aga Khan University

\section{Nadeemullah Khan}

Department of Emergency Medicine, Aga Khan University

\section{Munawar Khursheed}

Department of Emergency Medicine, Aga Khan University

\section{Junaid A. Razzak}

Department of Emergency Medicine, Aga Khan University 